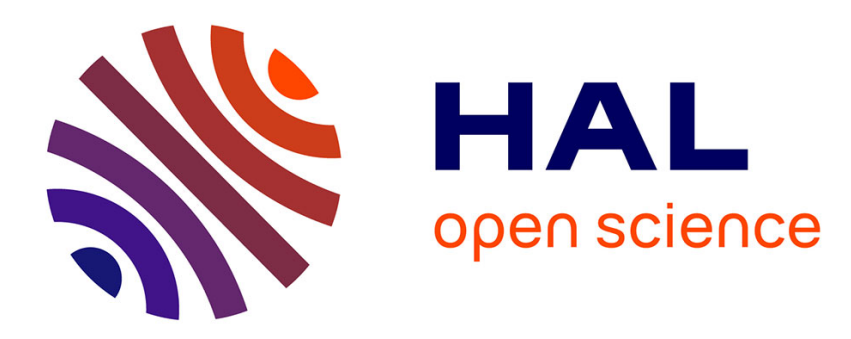

\title{
Germination en phase condensée et applications aux basses températures
}

\author{
D. Clausse
}

\section{To cite this version:}

D. Clausse. Germination en phase condensée et applications aux basses températures. Revue de Physique Appliquée, 1988, 23 (11), pp.1767-1772. 10.1051/rphysap:0198800230110176700 . jpa00246006

\section{HAL Id: jpa-00246006 https://hal.science/jpa-00246006}

Submitted on 1 Jan 1988

HAL is a multi-disciplinary open access archive for the deposit and dissemination of scientific research documents, whether they are published or not. The documents may come from teaching and research institutions in France or abroad, or from public or private research centers.
L'archive ouverte pluridisciplinaire HAL, est destinée au dépôt et à la diffusion de documents scientifiques de niveau recherche, publiés ou non, émanant des établissements d'enseignement et de recherche français ou étrangers, des laboratoires publics ou privés. 
Classification

Physics Abstracts

$07.20 \mathrm{M}-64.70 \mathrm{D}-82.70$

\title{
Germination en phase condensée et applications aux basses températures
}

\author{
D. Clausse \\ Département de Génie Chimique, DGTE, Université de Technologie de Compiègne, B.P. 233, 60206 \\ Compiègne, France
}

(Reçu le 11 avril 1988, accepté le 13 juillet 1988)

\begin{abstract}
Résumé. - Cet article décrit tout d'abord les caractéristiques de la nucléation d'une phase solide à partir d'une phase liquide surfondue. Les résultats expérimentaux obtenus sur des phases liquides surfondues dispersées sont donnés. Une méthode d'obtention de poudres par germination bloquée ou cristallisation des gouttes d'une émulsion est proposée.
\end{abstract}

\begin{abstract}
First, this article is a study of the characteristics of the nucleation of a solid phase from an undercooled liquid. Experimental results obtained by studying undercooled dispersed liquids within an emulsion are given. A procedure for obtaining powders through stopped germination or crystallization of droplets dispersed within an emulsion is proposed.
\end{abstract}

Cet article décrit la partie traitant de la germination en phase condensée et des applications en cryogénie telles que la formation de poudres, présentée dans l'exposé intitulé « Equilibres et germination de phases aux basses températures ».

\section{Cinétique de nucléation.}

La formation d'une phase solide à partir d'une phase liquide surfondue est déclenchée par un phénomène de nucléation. Cette interprétation apparaît lorsque l'on étudie [1] d'un point de vue quantitatif l'équilibre thermodynamique d'une goutte d'eau de rayon $a$ contenant une particule de glace assimilée à une sphère de rayon $r$, le tout placé dans de l'air humide. Ainsi à partir de l'équation de Kelvin traduisant cet équilibre on peut tracer les variations de la température d'équilibre du système en fonction du rayon de la particule de glace pour un diamètre donné de la goutte d'eau. Ainsi pour $a=100 \mu \mathrm{m}$, on obtient la figure 1.

On constate qu'il faudrait atteindre des températures $T$ très basses (valeur de $T$ pour $r \rightarrow 0$ ) pour réaliser l'équilibre d'une phase solide microscopique au sein de la phase liquide surfondue. Or on constate expérimentalement que les températures de solidification de gouttes d'eau surfondue sont supérieures à $-40^{\circ} \mathrm{C}$. Il faut donc envisager que la formation d'une nouvelle phase ne commence pas de façon continue mais se produit plutôt de façon spontanée

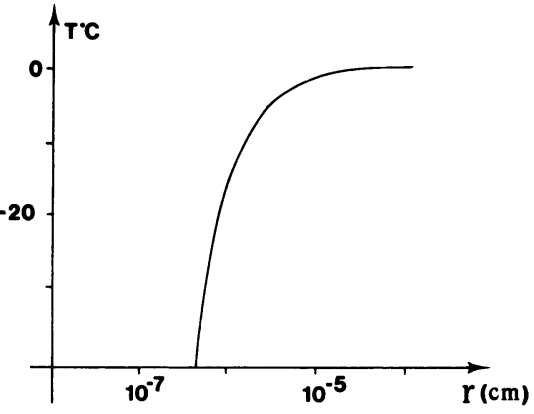

Fig. 1. - Température à l'équilibre d'une gouttelette d'eau de rayon $=100 \mu \mathrm{m}$, comportant une particule de glace sphérique dont le rayon est $r(\mathrm{~cm})$.

[Equilibrium temperature of a water droplet radius $100 \mu \mathrm{m}$, including a spherical ice particle whose radius is $r$ (cm).]

comme conséquence des fluctuations de densité et de température au sein du système métastable. Les aggrégats résultant de l'agglomération aléatoire de molécules sont généralement désignés « embryons". De tels embryons possèdent de faibles énergies de liaisons et se désagrègent aisément du fait de l'agitation thermique. Cependant à une température $T$, certains embryons atteignent une dimension critique et sont en équilibre instable avec la phase mère. Ces embryons de taille critique appelés «germes" vont 
croître spontanément si, en conséquence des fluctuations au sein de la phase mère, leur taille augmente d'une quantité infinitésimale. La compréhension du phénomène de nucléation passe donc par la connaissance de la distribution des embryons. Dans cette analyse [2] on considère une population d'embryons contenant $i$ molécules et désignés par $A_{i}$. On suppose qu'ils peuvent croître par la capture de monomères et que l'on a un état d'équilibre dynamique pour les embryons, soit :

$$
A_{i-1}+A_{1} \rightarrow A_{i} \quad(i=1,2, \ldots) .
$$

Par sommation de ces équations écrites pour les différentes valeurs de $i$, on obtient :

$$
i A_{1} \rightarrow A_{i}
$$

Cette relation traduit un équilibre qui est caractérisé par l'équation d'équilibre :

$$
i \mu_{1}=\mu_{i}
$$

où $\mu_{i}$ est le potentiel chimique du constituant $i$. Appelant $\Delta \Phi_{i}$ l'énergie de formation d'un embryon de taille $i$, le nombre d'embryons $N_{i}$ par unité de volume et correspondant à cette taille est exprimé en termes d'une distribution de Boltzmann, soit :

$$
n_{i}=n_{\mathrm{L}} \exp \left[-\Delta \Phi_{i} / k T\right]
$$

$n_{\mathrm{L}}$ étant le nombre de molécules par unité de volume de la phase liquide.

Supposant l'embryon de taille $i$ assimilable à une sphère de rayon $r$, on peut écrire $\Delta \Phi_{i}=\Delta \Phi(r)$ sous la forme

$$
\Delta \Phi=-A r^{3}+B r^{2}
$$

Le premier terme correspond à l'énergie libérée lorsque les molécules ou atomes s'associent pour former des liaisons chimiques. $A$ peut être considéré comme un paramètre traduisant la cohésion entre particules de l'embryon. Ce terme rend donc compte des forces moléculaires favorisant la nucléation. Le second terme provient de l'énergie à fournir pour former une nouvelle surface. $B$ est un paramètre fonction de la tension interfaciale solide-liquide, $\sigma$, et le produit $B r^{2}$ représente donc les forces œuvrant contre la nucléation. L'expression complète de $\Delta \Phi$ à une température donnée est obtenue en écrivant que $\Delta \Phi$ représente la différence du potentiel thermodynamique du système entre les états correspondant au liquide surfondu seul et au liquide surfondu comprenant en son sein et en équilibre, un embryon de rayon $r$. La définition de la fonction $\Phi$ nécessite quelques précautions [3-5] du fait de l'existence d'une pression à l'intérieur de l'embryon, différente de celle existant à l'extérieur en raison des faibles dimensions de l'embryon. On obtient [6] :

$$
\Delta \Phi=-\frac{4}{3} \pi r^{3} n_{\mathrm{s}} k T \ln \frac{P_{\mathrm{L}}}{P_{\mathrm{s}}}+4 \pi \sigma r^{2}
$$

où $P_{\mathrm{L}}$ et $P_{\mathrm{s}}$ sont les pressions de vapeur saturée audessus des surfaces planes du liquide surfondu et du solide à la même température $T$ et $n_{\mathrm{s}}$ le nombre de molécules par unité de volume de la phase solidifiée. Les variations de $\Delta \Phi$ en fonction de $r$ sont représentées par une courbe qui est tangente à l'axe des abscisses en $\mathrm{O}(r=0)$, présente un maximum pour $r=r_{\mathrm{g}}$, et est limitée en $N$ (Fig. 2).

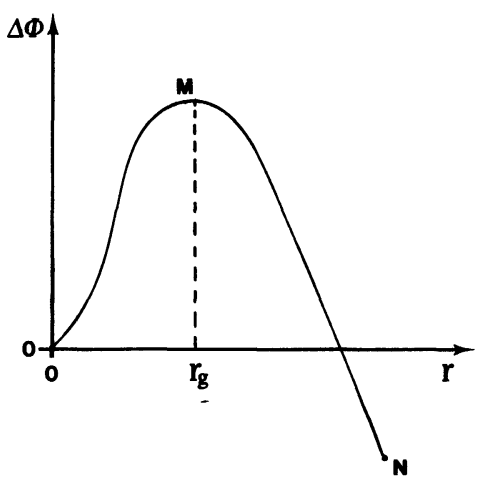

Fig. 2. - Energie de formation $\Delta \Phi$ d'un embryon de rayon $r$, en fonction de $r$.

[Energy of formation $\Delta \Phi$ of an embryo radius $r$, versus $r$.]

A la pression $P$ et la température $T$ du système le point $\mathrm{O}$ représente le liquide surfondu, le point $\mathrm{N}$ le système totalement solidifié et le point $M$ l'état d'équilibre entre la phase liquide surfondue et l'embryon de rayon $r_{\mathrm{g}}$ que l'on appelle germe. Cet état correspondant à un maximum de $\Delta \Phi$ nous indique que cet équilibre est instable et qu'en dehors de lui aucun embryon solide ne peut subsister en présence de la phase surfondue : un embryon de rayon $r<r_{\mathrm{g}}$ disparaît aussitôt formé, tandis qu'un embryon de rayon $r>r_{\mathrm{g}}$ grossit aux dépens de la phase surfondue, jusqu'à disparition totale de celleci.

La barrière d'énergie qui correspond à la formation d'un germe de rayon $r_{\mathrm{g}}$ est donnée par:

$$
\begin{array}{ll}
\frac{\mathrm{d}(\Delta \Phi)}{\mathrm{d} r}=0 & \text { soit } \Delta \Phi_{\mathrm{g}}=\frac{16 \pi \sigma^{3}}{3\left[n_{\mathrm{s}} k T \ln \frac{P_{\mathrm{L}}}{P_{\mathrm{s}}}\right]^{2}} \\
\text { avec } & \ln \frac{P_{\mathrm{L}}}{P_{\mathrm{s}}}=\frac{2 \sigma}{r_{\mathrm{g}}} \frac{1}{n_{\mathrm{s}} k T} \\
\text { et } & r_{\mathrm{g}}=\frac{2 \sigma}{n_{\mathrm{s}} \Delta H_{\mathrm{s}}(T)} \frac{T_{\mathrm{e}}}{T-T_{\mathrm{e}}} \\
\text { soit } & \Delta \Phi_{\mathrm{g}}=\frac{4}{3} \pi \sigma r_{\mathrm{g}}^{2}
\end{array}
$$


où $\Delta H_{\mathrm{s}}$ représente l'enthalpie molaire moyenne de solidification entre la température $T_{\mathrm{e}}$ de l'équilibre solide-liquide et la température $T<T_{\mathrm{e}}$.

La vitesse de nucléation $J_{0}$, c'est-à-dire le nombre de germes formés, en régime stationnaire, par unité de temps et de volume liquide correspond à la vitesse avec laquelle les germes vont capter une molécule supplémentaire afin de pouvoir franchir la barrière d'énergie $\Delta \Phi_{g}$ et donc croître librement. Cette vitesse est donnée par :

$$
J_{0}=A \exp \left[-\frac{\Delta \Phi_{\mathrm{g}}}{k T}\right]
$$

où le facteur préexponentiel est un terme complexe faisant intervenir un terme exponentiel de la forme : $\exp \left(-\Delta \Phi^{\prime} / k T\right), \Delta \Phi^{\prime}$ représentant l'énergie d'activation $E$ du transfert d'une molécule à travers l'interface liquide-solide.

La valeur numérique $J_{0}(T)$ ne peut être obtenue à partir de l'expression (8) en raison de nombreux paramètres inconnus dans cette équation comme par exemple la tension interfaciale $\sigma$. Comme $\sigma$ apparaît à la puissance trois dans $\Delta \Phi_{\mathrm{g}}$ (relation 4) une variation de $10 \%$ de $\sigma$ entraîne une modification de $J_{0}$ d'un facteur $10^{6}$. Cependant on peut prévoir l'allure des variations de $J_{0}(T)$ représentée figure 3 .

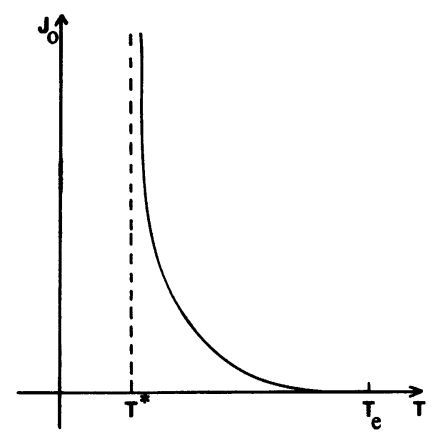

Fig. 3. - Variations de la vitesse de nucléation $J$ en fonction de la température $T . T_{\mathrm{e}}$ : température à l'équilibre solide-liquides $T^{*}$ : la température la plus probable du seuil de rupture de surfusion.

[Changes of the nucleation rate $J$ versus temperature $T$. $T_{\mathrm{e}}$ : equilibrium solid-liquid temperature, $T^{*}$ : the most probable temperature of the breakdown of the undercooling.]

Dans le cas d'un liquide présentant une viscosité importante les variations de $J_{0}$ en fonction de $T$ sont modifiées. La viscosité $\eta$ est reliée à $\Delta \Phi^{\prime}=E$ selon la relation

$$
\eta=\eta_{0} \mathrm{e}^{\frac{E}{k T}}
$$

Il apparaît que plus un liquide est visqueux plus faible sera la vitesse de nucléation $J_{0}$. Si l'on suppose
$E$ constant on peut voir qu'au cours d'un refroidissement les termes $\frac{E}{k T}$ et $\frac{\Delta \Phi_{\mathrm{g}}}{k T}$ varient en sens inverse.

En effet si $T$ diminue $E / k T$ augmente alors que $\frac{\Delta \Phi_{\mathrm{g}}}{k t}$ diminue car $\Delta \Phi_{\mathrm{g}}$ est proportionnel à $1 /\left(T_{\mathrm{e}}-T\right)^{2}$ terme prépondérant (relations 6 et 7 ). On en déduit dans ce cas les variations de $J_{0}$ en fonction de $T$ représentées figure $4 . J_{0}$ présente alors une valeur maximum que nous désignerons par $J_{0}^{\mathrm{Max}}$.

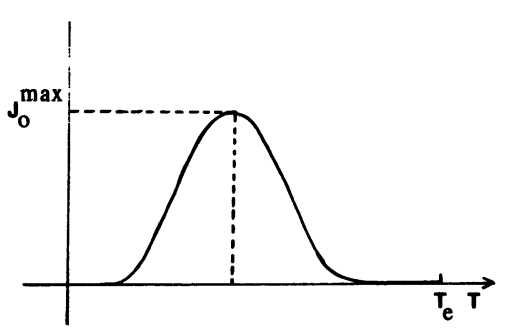

Fig. 4. - Vitesse de nucléation en fonction de la température pour un liquide présentant une forte viscosité.

[Nucleation rate versus temperature for a liquid showing high viscosity.]

\section{Probabilité de nucléation.}

Les caractéristiques du phénomène de nucléation entraînent que considérant un échantillon on peut uniquement définir une probabilité de nucléation et donc de solidification alors que la fusion s'effectue elle à la température $T_{\mathrm{e}}$ quelque soit l'échantillon considéré.

Il existe une relation entre la probabilité de nucléation, le volume du liquide surfondu, le temps et le degré de surfusion $\Delta T=T_{\mathrm{e}}-T(7)$. La probabilité de nucléation se produisant dans un volume $V$ dans l'intervalle de temps $t, t+\mathrm{d} t$ est donnée par :

$$
V J_{0}(T) \mathrm{d} t
$$

$J_{0}$ apparaît comme étant la probabilité d'apparition d'un germe dans un échantillon de volume unité observé pendant une unité de temps tant que $J_{0}^{\mathrm{Max}} \leqslant 1$. On voit l'importance qu'il y a à connaître si $J_{0}^{\max }$ est inférieur à 1 ou non et suivant les valeurs de $E$ donc de la viscosité on peut avoir les deux cas représentés figure 5 .

Dans le cas (a) on peut affirmer qu'il existe une température pour laquelle un volume unité aura cristallisé en une unité de temps. Dans le cas (b) on peut ne pas observer de nucléation donc de solidification dans tout le domaine de température où la probabilité est différente de zéro.

Considérant une population de $N_{0}$ échantillons de même volume $V$, on peut déduire de cette analyse que tous les échantillons ne seront pas nucléés à la 


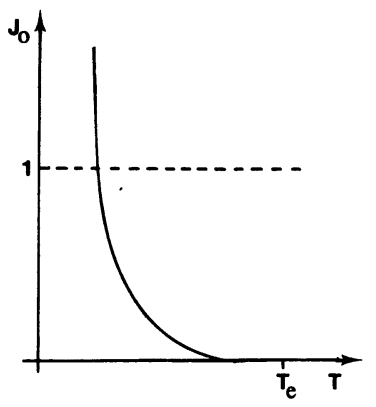

a)

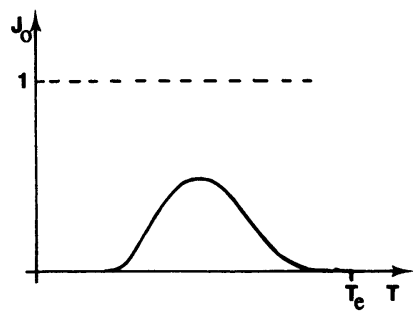

b)
Fig. 5. - Corrélation entre la probabilité de cristallisation et la viscosité d'un liquide surfondu.

[Correlation between cristallization probability and viscosity of an undercooled liquid.]

même température au cours d'un refroidissement continu ou au même instant lors de la stabilisation à une température $T$. Si $P(V, t)$ est la probabilité de nucléation d'un échantillon dans l'intervalle de temps $(0, t)$ alors :

$$
P(V, t)=\frac{N_{t}}{N_{0}} .
$$

$N_{t}$ étant le nombre d'échantillons solidifiés à l'instant $t$. Dans cette analyse on considère qu'il suffit d'un germe pour entraîner la solidification totale de l'échantillon. Le nombre d'échantillons nucléés dans l'intervalle $t, t+\mathrm{d} t$ est donné par

$$
N_{t+\mathrm{d} t}=N_{t}+\left(N_{0}-N_{t}\right) V J_{0} \mathrm{~d} t
$$

Soit en divisant par $N_{0}$

$$
\begin{gathered}
P(V, t+\mathrm{d} t)=P(V, t)+[1-P(V, t)] V J_{0} \mathrm{~d} t \\
\text { d'où } \quad \frac{\partial P(V, t)}{\partial t}=[1-P(V, t)] V J_{0}(T) \mathrm{d} t
\end{gathered}
$$

soit par intégration

$$
\ln [1-P(V, t)]=-\int_{0}^{t} V J_{0}(T) \mathrm{d} t .
$$

Deux cas sont à considérer :

$-N_{0}$ échantillons sont maintenus à une température fixe $T<T_{\mathrm{e}}$. L'équation (14) devient :

$$
\ln [1-P(V, t)]=-V t J_{0}(T)
$$

- les $N_{0}$ échantillons sont soumis à un refroidissement continu à la vitesse $\dot{T}=\frac{\mathrm{d} T}{\mathrm{~d} t}$. L'équation (14) s'écrit :

$$
\ln [1-P(V, t)]=-\frac{V}{\dot{T}} \int_{T_{\mathrm{e}}}^{T} J_{0}(T) \mathrm{d} T
$$

$T$ étant la température atteinte au cours du refroidissement.

On voit donc selon les formules (15) et (16) que plus grand sera le volume $V$ des échantillons, plus court sera le temps à attendre pour observer la solidification à une température donnée et plus proche de $T_{\mathrm{e}}$ sera la température de solidification lors d'un refroidissement continu. De même, plus grande sera la vitesse de refroidissement, plus faible sera la probabilité de solidification à une température donnée. En fait, l'amplitude de ces phénomènes est fonction des valeurs de $J_{0}$ en fonction de $T$. Si $J_{0}$ est supposé pratiquement nulle pour $T>T^{*}$ (cf. Fig. 3) alors on ne verra pratiquement pas d'effet de volume et les échantillons placés à une température $T$ différente de $T^{*}$ ne se solidifieront pas dans un temps à l'échelle humaine.

\section{Résultats expérimentaux et applications.}

De nombreuses études ont été réalisées notamment sur des émulsions, la phase dispersée représentant un ensemble de $N_{0}$ échantillon placés dans les mêmes conditions [8-9]. L'analyse des thermogrammes obtenus lors d'un refroidissement et réchauffement continus (Fig. 6) d'une émulsion d'eau révèlent une solidification des gouttes entre $-38^{\circ} \mathrm{C}$ et $-42^{\circ} \mathrm{C}$ avec un maximum de cristallisation à
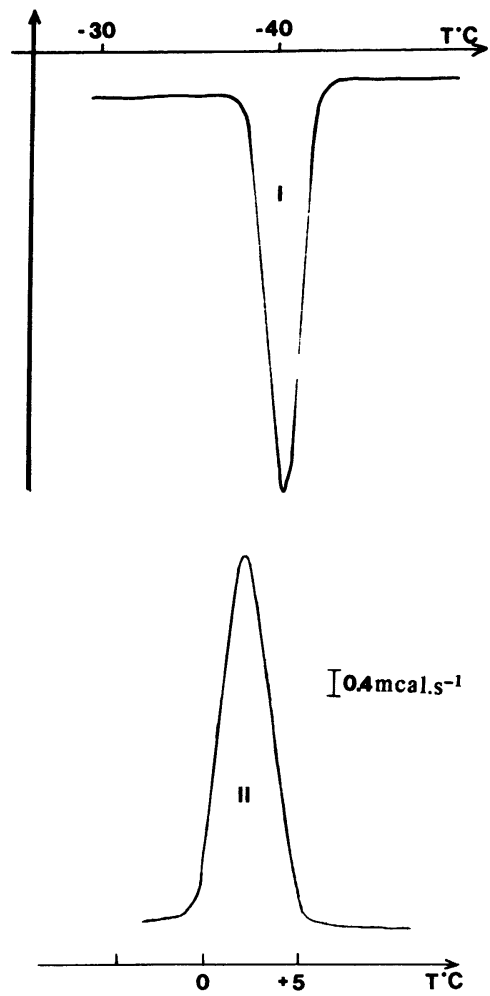

Fig. 6. - Thermogrammes de refroidissement (I) et de réchauffement (II) d'une émulsion d'eau.

[Cooling (I) and heating (II) thermogrammes of a water emulsion.] 
$T^{*}=-40^{\circ} \mathrm{C}$ et une fusion des gouttes solidifiés à $0{ }^{\circ} \mathrm{C}$ pour toutes les gouttes [10]. Ces études réalisées sur des émulsions dont la phase dispersée est une solution aqueuse de $\mathrm{NH}_{4} \mathrm{Cl}$ ont permis de compléter le diagramme de phases du binaire considéré par des courbes d'hypercongélation $(\Gamma)$ et d'hypersolubilité $\Sigma$ lieu des températures de rupture de surfusion $(\Gamma)$ ou de rupture de sursaturation $(\Sigma)$ des gouttes dispersées [4-11] (Fig. 7).

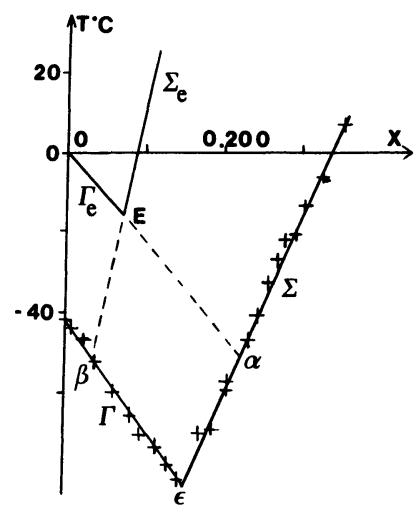

Fig. 7. - Courbes d'hypercongélation $(\Gamma)$ et d'hypersolubilité $(\Sigma)$ de solutions aqueuses de chlorure d'ammonium dispersées dans des émulsions.

[Hyperfreezing $(\Gamma)$ and hypersolubility $(\Sigma)$ curves of aqueous solutions of ammonium chloride dispersed within emulsions.]

Des études de cinétique de solidification à température fixe ont montré que la phase dispersée pouvait se solidifier en des temps relativement courts. Une possible nucléation à l'interface Eau/Huile a été considérée pour expliquer les phénomènes observés [9-10].

L'obtention de poudres peut s'effectuer par réalisation d'une germination bloquée. La formation d'une nouvelle phase résulte en fait de deux mécanismes :

- formation d'un germe,

- développement du germe.

Le développement du germe est contrôlé par la diffusion des molécules dans la phase mère. On observe une germination bloquée si la viscosité du milieu est trop importante pour permettre la croissance du germe. Les vitesses relatives de ces mécanismes déterminent la taille des particules formées. L'obtention de systèmes quasi-monodispersés est réalisée lorsque la nucléation se fait sur une période relativement courte au début de la formation des particules. Ceci peut être réalisé en ensemençant la phase liquide métastable avec de très petites particules ou en réalisant une brève nucléation homogène par refroidissement rapide. C'est ainsi que l'on peut former des solutions colloïdales d'iodure d'argent.

La réalisation d'émulsions peut aussi permettre le contrôle de la taille des particules formées. On peut concevoir un processus qui comprendrait les différentes étapes suivantes :

1) formulation de l'émulsion : recherche du surfactant et du milieu continu les plus appropriés compte tenu de la nature de la poudre que l'on veut former et donc du matériau liquide que l'on veut disperser ;

2) analyse de l'émulsion : définition de la polydispersité qui correspond à celle de la poudre formée si on néglige les variations de volume lors de la solidification ;

3) étude de la nucléation : cristallisation des gouttes dispersées. Cette analyse peut se faire par calorimétrie (détection de l'enthalpie libérée lors de la solidification des gouttes) ou conductivimétrie si le système présente une différence de conductivité décelable lors du changement d'état;

4) Récupération des granules formés.

Signalons pour terminer la possibilité de réaliser une réaction chimique entre les phases dispersées de deux émulsions, réaction qui peut conduire à la formation d'un précipité sous forme divisé puisqu'il se trouve dispersé dans les gouttes de l'émulsion résultant du mixage.

Cette technique a été utilisée pour former de l'iodure d'argent solide dispersé [11]. La réaction chimique a été réalisée par mélange de deux émulsions dont la phase dispersée de l'une est une solution aqueuse de $\mathrm{AgNo}_{3}$ et celle de l'autre une solution aqueuse de $\mathrm{KI}$.

\section{Bibliographie}

[1] Pruppacher, H. R. and Klett, J. D., Microphysics of clouds and precipitation (D. Radel Publishing Company) Dordrecht : Holland, Boston : U.S.A. or London : England (1978) p. 149.

[2] Même référence que [1] p. 162.

[3] Abraham, J. Atm. Sci. 25 (1968) 47-53.

[4] Clausse, D., Thèse de Doctorat ès Sciences Physiques, Pau (1972).

[5] Clausse, D., J. Chim. Phys., Abrégé (1976) 76-1.
[6] Zettlemayer, A. C., Nucléation (Marcel Dekker) N.Y. (1969) p. 463.

[7] Carte, A. E., Proc. Phys. Soc., London 73 (1959) 324.

[8] Clausse, D., Encyclopedia of Emulsion Technology, Ed. P. Becher (Marcel Dekker) N.Y. (1985), vol. 3, chap. 2, pp. 77-157.

Ce chapitre est une revue des techniques de recherche mettant en jeu des émulsions. Le lecteur trou- 
vera dans ce chapitre de nombreuses références d'articles traitant des phénomènes de nucléation. dans les émulsions.

[9] Clausse, D., Dumas, J. P., Meijer, P. H. E., Broto, F., J. Disp. Sci. Tech. 8 (1987) 1-28.
[10] Clausse, D., Babin, L., Broto, F., Aguerd, M. et Clausse, M., J. Phys. Chem. 87 (1983) 4030.

[11] Aguerd, M., Broto, F., Babin, L. et Clausse, D., Coll. Surf. 12 (1984) 33-340. 